\title{
Towards Implementing Framework to Generate Myopathic Signals
}

\author{
Amira Dridi ${ }^{1 *}$, Jassem Mtimet ${ }^{2 *}$, Slim Yacoub ${ }^{3 *}$ \\ *Signal, Image and Technology of Information Laboratory, National Engineering School of Tunis \\ Tunis el Manar University, BP 37 Belvedere, 1002, Tunis, Tunisia
}

\begin{abstract}
In this paper, we describe a simulation system of myopathicsurface electromyography (sEMG) signals. The architecture of the proposed system consists of two cascading modules. SEMG signals of three pathological skeletal muscles (Biceps Brachii, InterosseursDorsalis, TibalisAnterior) were generated. Root Mean Square (RMS Envelope) and Power Spectral Density (PSD) were used to validate our system.
\end{abstract}

Keywords-Component; Surface Electromyography (sEMG); myopathy; root mean square; Power Spectral Density (PSD; skeletal muscles; biceps brachii; interosseous dorsalis; tibialis anterior

\section{INTRODUCTION}

Electromyography covers the study of muscle function through electrical signals. This medical examination collects measures and records the electrical signal that propagates in the nerves or in the muscle fibers (Action Potential). It consists of plotting the variations of the muscular membrane on the display screen; this diagnostic procedure is performed either in a non-invasive manner using skin contact electrodes (surface electromyography) or in an invasive manner using needle electrodes (invasive electromyography). These detection processes are often used in several fields such as: neuromuscular clinical diagnostics, rehabilitation, prosthesis control, muscle fatigue studies and gait analysis [1-4].

The mathematical modeling of surface electromyography (sEMG) is a method which allows to synchronize physiological parameters (e.g. recruitment frequency, conduction rate...) with simulated results in order to analyze their influences and to test the validity of the algorithms used to process this kind of signals [5-7]. Recently, research studies have focused on different approaches to modeling and to simulating sEMG signals, which are based on phenomenological as well as physiological aspect [8-10]. In [1] and [6], the authors propose an in-depth recapitulative study of these approaches.

Myopathic diseases are disorders in which skeletal muscle is mainly involved. Several factors can cause myopathies including inherited genetic defects (e. g. muscular dystrophies), endocrine, inflammatory or metabolic abnormalities. The different myopathies lead weakness and atrophy of skeletal muscles. Other symptoms of myopathy include fatigue, stiffness, and muscle cramps [11].

Some myopathies, such as muscular dystrophies, develop very early, while others develop later in patient life. Some of them gradually worsen over time and do not respond well to treatment, while others appear treatable and often remain stable for long periods of time [1].

There are no several studies interested to model this kind of signals. However, their generation provides a significant contribution in several areas. For example, for classification purposes, a clinical study is required to build a classification model that is costly in terms of time and resources. In the interest of processing these signals, we propose a model that can be used to generate myopathic signals for different types of skeletal muscles.

The paper will be organized as follows: Section 2 presents the components of the myopathicsEMG signal generation system. Section 3 illustrates the experimental results of the proposed simulation model. Finally, in Section 4 we close with a brief conclusion.

\section{MATERIALS AND METHODS}

The physiological and anatomy studies of striated skeletal muscles reveal their composition in motor units (MU), which are composed of motoneurons and muscle fibers. In this section, we present a mathematical-based model which generates the electrical activity of myopathic muscular pathologies. The below diagrams (Fig. 1 and Fig. 2) represent the different components of our generation model.

\section{A. Intracellular Action Potential Generation}

The generation of the intracellular action potential (IAP) produces a transmembrane ionic current $I_{m}(t)$ that propagates along the outer membrane of muscle fiber (sarcolemma). Moreover, the fiber is considered as a propagation tube for axially circulating current [9]. We use the following formula to generate the aforementioned current [17]:

$I_{m}(t)=$

C.A. $(\lambda v)^{2} \cdot(\lambda \cdot v \cdot t) \cdot\left(6-6 * \lambda \cdot v \cdot t+\lambda \cdot v^{2} \cdot t^{2}\right) \cdot e^{-\lambda v t}$

With:

- A, C: constants affecting the amplitude of $\mathrm{I}_{\mathrm{m}}$

- $\lambda$ : Scale factor for adapting the model to the real observations

- $\quad \mathrm{v}$ : speed of current propagation along the fibers

Consequently, myopathic IAPs characterizing by a short duration and low amplitude are produced after a values modification in the responsible parameters of this phenomenon $(\mathrm{A}, \lambda)$. 


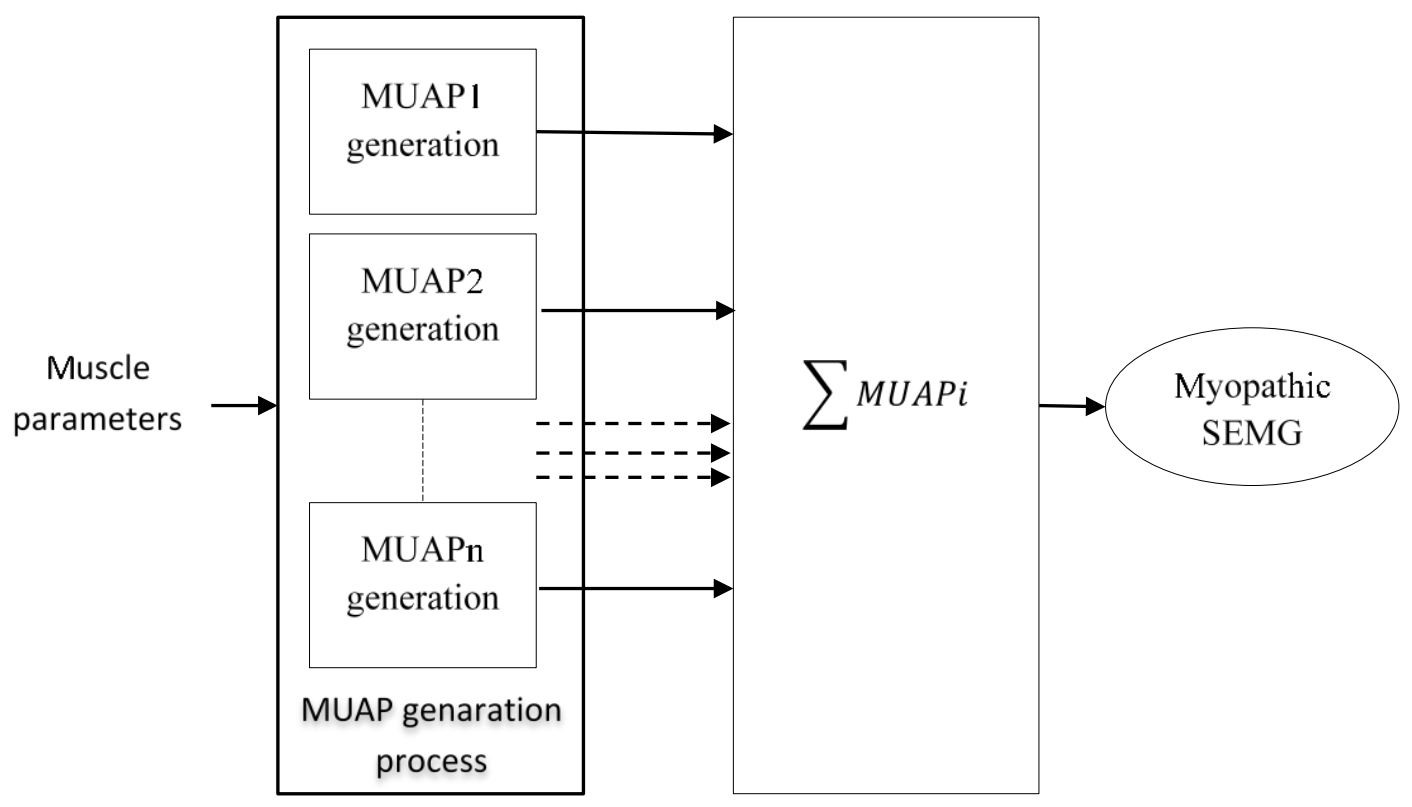

Fig. 1. Simulation Process of Myopathic SEMG.

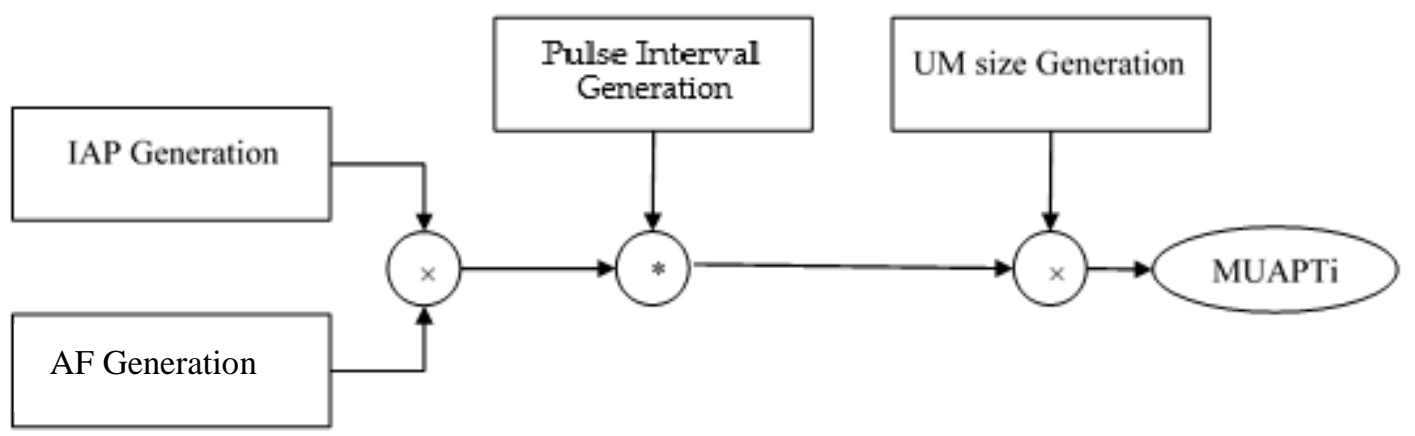

Fig. 2. Generation Process of MUAPi.

\section{B. Maintaining the Integrity of the Specifications}

When the action potential (AP) propagates along the muscle fiber, it is automatically attenuated. In this subsection, we develop the process used to generate the appropriate attenuation function (AF) for myopathic signals. The mitigation equation is as follows [18]:

$f=\frac{1}{4 . \pi \partial_{e}} \frac{1}{\sqrt{(z-z 1)^{2}+\partial_{e}\left((x-x 1)^{2}+(y-y 1)^{2}\right)}}$

Such as:

- $(\mathrm{x}, \mathrm{y}, \mathrm{z})$ the origin coordinates

- $(\mathrm{x} 1, \mathrm{y} 1, \mathrm{z} 1)$ electrode coordinates

- $\partial_{\mathrm{e}}$ : media conductivity

Y1 represents the distance between the muscle fiber and the detection electrode. Typically, it's a random value between $33.10^{-03}$ and $37 \cdot 10^{-03} . \partial_{\mathrm{e}}$ represents the conductivity value of the medium. In the case of myopathic patient, the extracellular medium is characterized by a high conductivity compared to the normal one. For this purpose, we multiply the value of $\partial_{\mathrm{e}}$ by five.

\section{Generation of Pulse Intervals}

The MU discharge phenomenon is an essential process to generate SEMG signals. It depends on the inter-pulse interval (IPI) which represents the time interval between two successive pulses. Furthermore, this process is activated by exciting the motor unit $\mathrm{MU}_{\mathrm{i}}$ at the randomly defined moment $\mathrm{t}(\mathrm{i})$.

We suppose both the last excitation moment $t_{i(j-1)}$ and the firing rate $(\mathrm{FR})$ of $\mathrm{MU}_{\mathrm{i}}$ are known, we can then calculate the next excitation moment $t_{\mathrm{ij}}$.

In order to simulate this process, we assume that firing rates follow a random truncated Poisson distribution between 8 and $42 \mathrm{~Hz}$. 
Then, the excitation moment $t_{i j}$ is determined using the following equation:

$t_{i j}=t_{i(j-1)}+I P I_{i}$

Where: $I P I_{i}=\frac{1}{F R_{i}}$

\section{Structures Generation of Myopathic Motor units}

Skeletal muscles, commonly composed of $\mathrm{n}$ motor units (MU) having different mechanical as well as electrical characteristics that vary according to their size. Whereas, the MU size is measured terms of the number of muscle fibers it contains.

In myopathic cases, the number of UMs composing the muscle remains unchanged; however, a reduction in their sizes is identified according to an affectation percentage.

In order to generate the structure of Myopathic UM, we use the following random process:

MUsize $_{i}=k m_{i}-\left(k_{i} \times\right.$ affect $\left.\%\right)$

Such as: $\mathrm{km}_{\mathrm{i}}$ presents a random uniform distribution of number of fibers in normal $\mathrm{UM}_{\mathrm{i}}$ and $\mathrm{I}=[1 \ldots \mathrm{n}]$.

\section{E. Generation of SEMG}

As we know, this step takes into consideration the muscle physiology, the conductor volume and the detection system. Whereas, SEMG signal recorded using a single monopolar electrode may be considered as a superposition of $\mathbf{M}$ motor unit action potentials located at different depths under the human skin and activated in semi-random manner.

$\operatorname{SEMG}\left(Z_{A}, t\right)=\sum_{m=1}^{M} \operatorname{MUAP}_{m}(t)$

When the detection system is bipolar, resulting signal is obtained using the difference between the SEMG recorded by two monopolar electrodes located in positions $\mathrm{Z}_{\mathrm{A}}$ and $\mathrm{Z}_{\mathrm{B}}$.

$\operatorname{SEMG}(t)=\operatorname{SEMG}\left(Z_{A}, t\right)-\operatorname{SEMG}\left(Z_{B}, t\right)$

\section{RESULTS}

Using the simulation model presented in the previous section, we simulate normal and myopathic signals. We assume that the used detection system is differential with two parallel placed electrodes. The following figure (Fig. 3) shows two illustration examples (normal and myopathic with a loss percentage equal to $50 \%$ ). We can observe that signal relating to the muscle with anomaly presents a decrease in the amplitude and the duration of the MUAP.

Then, we focused on generating myopathic signals of three different skeletal muscles:

- Biceps brachii

- Interosseurs dorsalis

- Tibalisanterior

The composition of each muscle is described in Table I [12-14]. The obtained result is shown in Fig. 4.
For comparison purposes, we investigated the RMS factor (Fig. 5) as well as the PSD variation (Fig. 7-9) of the previously simulated signals $[15,16]$.

For each simulated signal, the RMS value where calculated. Given the SEMG signal S(j), the RMS value is defined as:

$R M S=\sqrt{\frac{1}{N} \sum_{j=1}^{N} S(j)^{2}}$

With $\mathrm{N}$ represents the number of samples.

We performed a boxplot to analyze the RMS values obtained from the simulated signals of the different muscles. Fig. 6 shows the aforementioned boxplot; it represents six analyzed signals corresponding to three normal muscles and three myopathic muscles.

As we can see, there are significant differences between the mean values of RMS for the three simulated signals, corresponding to the normal muscles. Moreover, the RMS of myopathic muscles are substantially different from the normal one.

The result showed significant decrease in mean RMS from $146.10-4$ to 3,6 . $10-4$, from 5. $10-5$ to $112,5.10-5$ and from 9,25. $10-5$ to $327.10-5$ at the biceps brachii, the interosseous dorsalis and the tibialis anterior, respectively muscles.
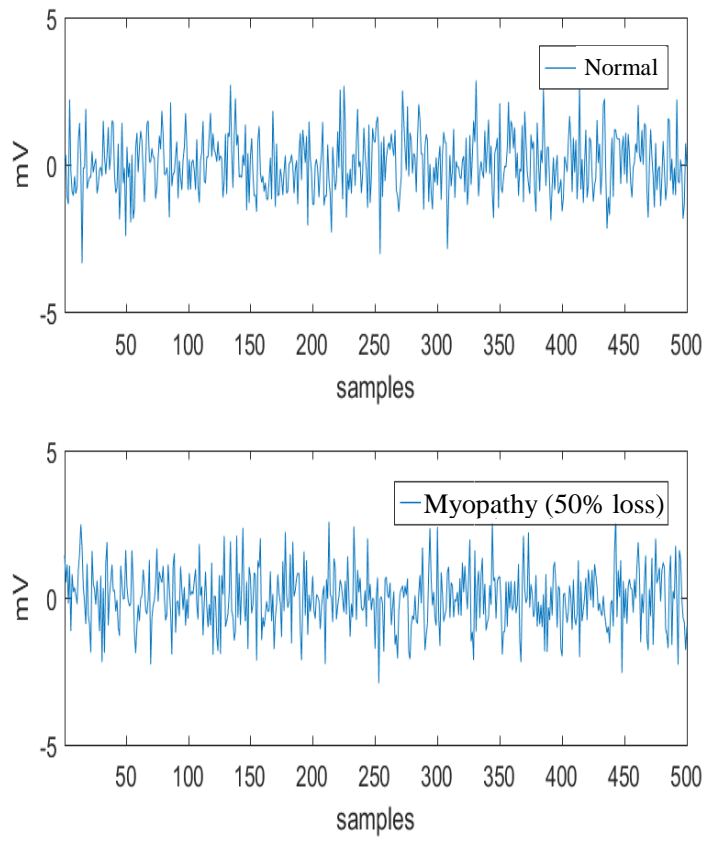

Fig. 3. Healthy and Myopathic SEMG Simulation Results.

TABLE I. SKELETAL MUSCLES COMPOSITION

\begin{tabular}{|l|c|c|}
\hline Type of muscle & MU number & $\begin{array}{l}\text { Number of } \\
\text { fibers /UM }\end{array}$ \\
\hline Biceps brachii (BB) & 774 & $750 \pm 50$ \\
\hline Interosseousdorsalis (IDA) & 119 & $340 \pm 50$ \\
\hline Tibialis anterior (TA) & 445 & $270 \pm 50$ \\
\hline
\end{tabular}



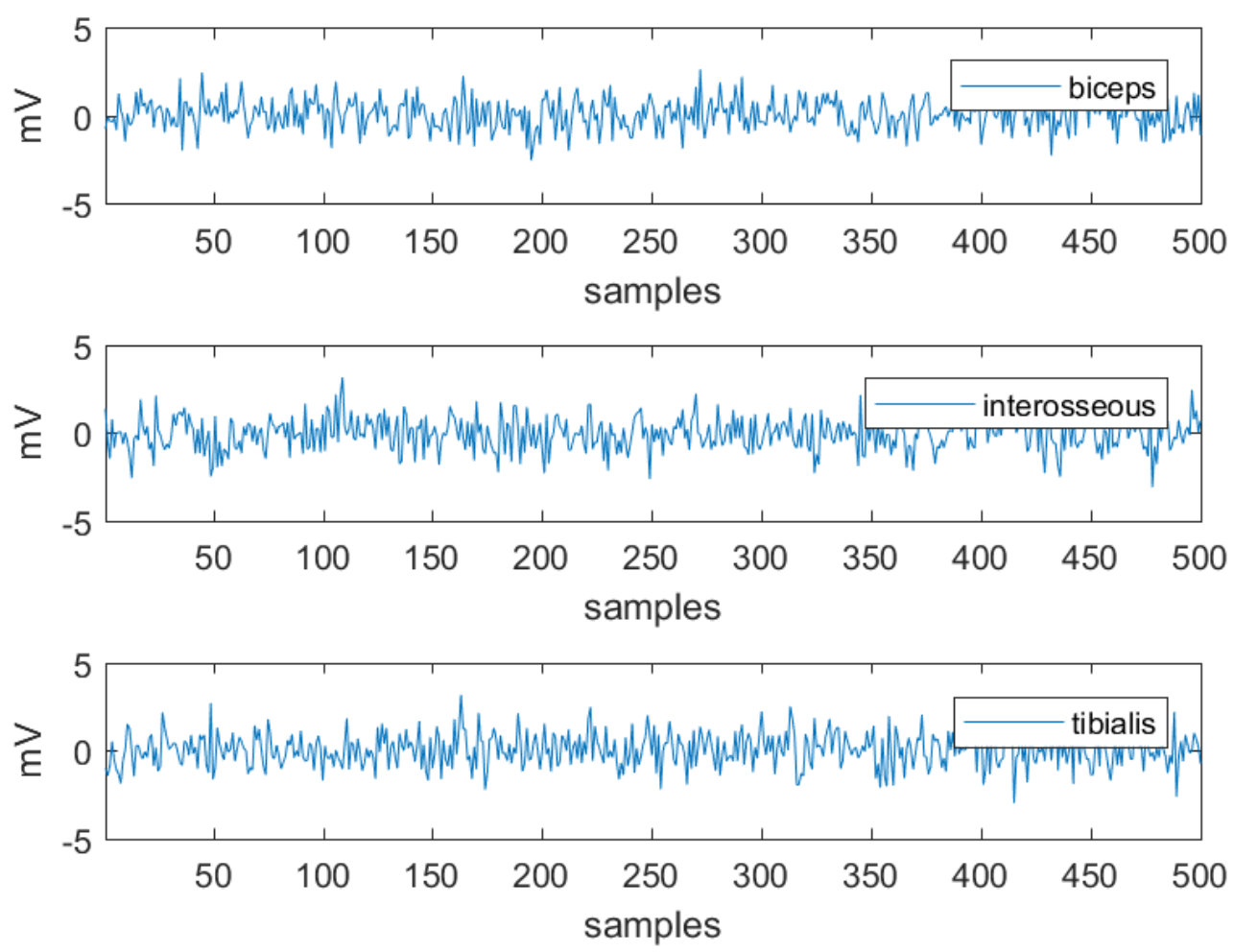

Fig. 4. Myopathic SEMG for different Muscles.
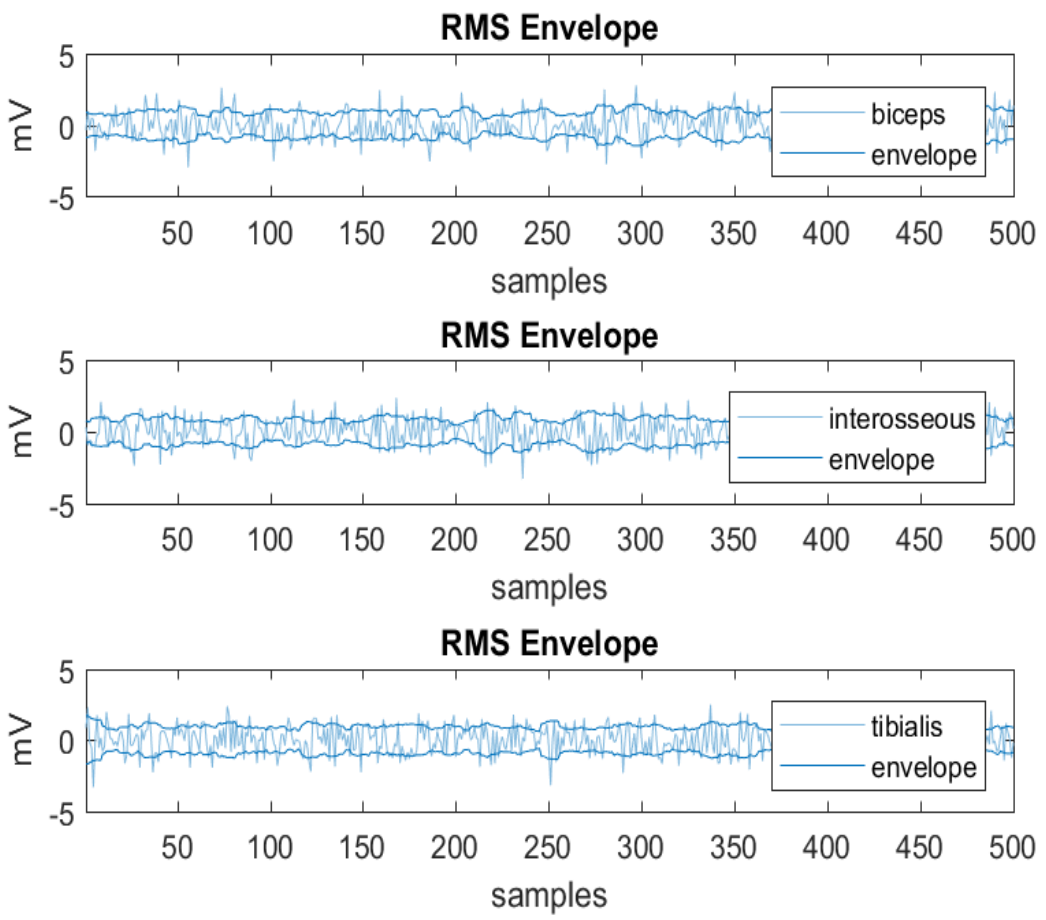

Fig. 5. RMS Envelope Results. 

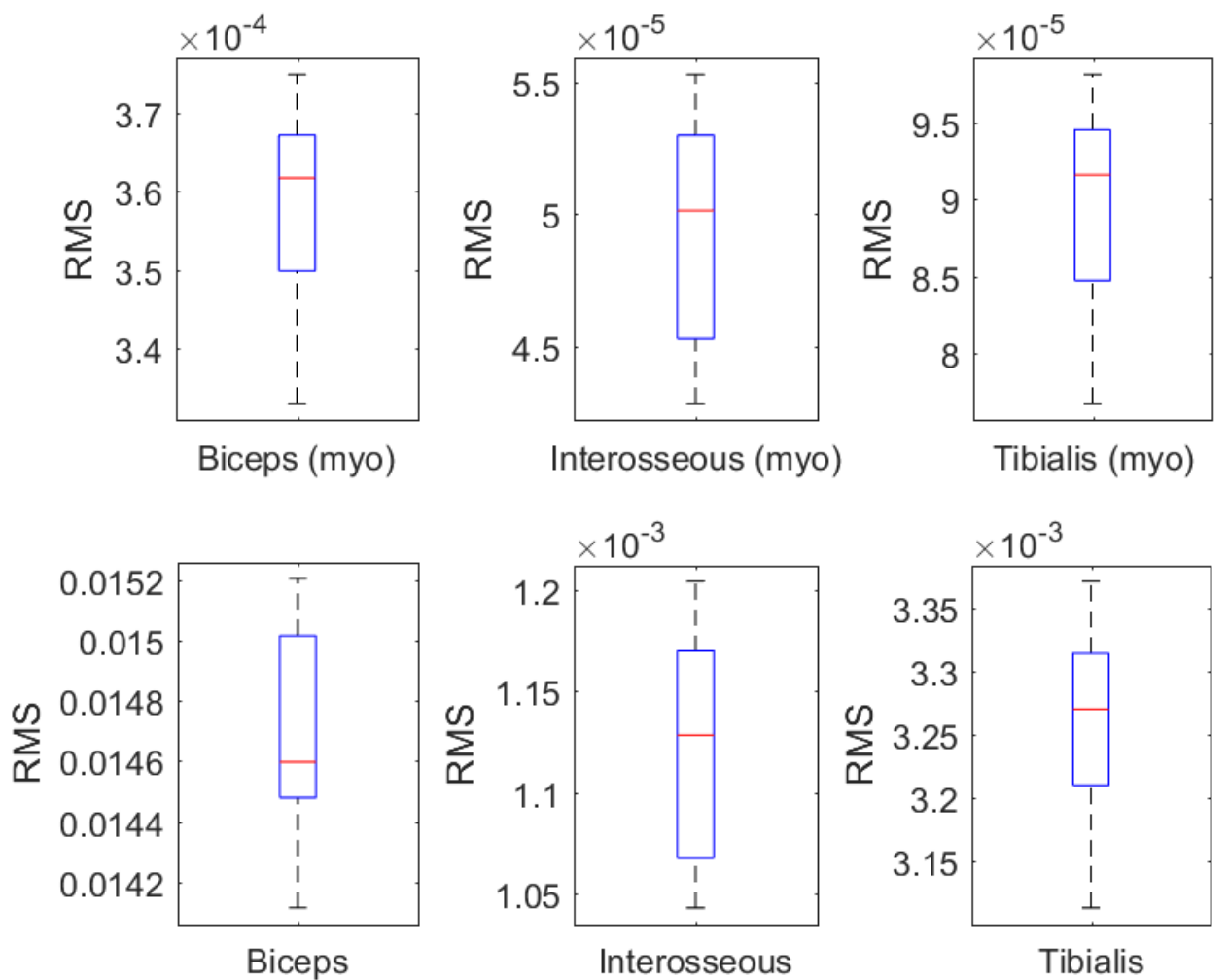

Tibialis

Fig. 6. Box Plot of RMS

In order to evaluate the electrical activity of the obtained signal we calculate their peak frequency using the above equation:

$P K F=\max \left(P S D_{i}\right)$

Where $\mathrm{PSD}_{\mathrm{i}}$ denote the SEMG power spectrum at frequency bin $i$ and $i=1 \ldots N$.

The boxplots in Fig. 10 illustrates the variation in the values of the PKF of different simulated signals. Based on the obtained results, we remark that all PKF of myopathic muscles is lower than those of normal muscles.

The peak frequency of myopathic patients' SEMG signals was significantly lower in tibialis than in other muscles (9.9 10-7 (TA) vs. 1.25 10-7 (IDA) and 1.9 10-5 (BB)).

As shown in Fig. 6-8, the simulated myopathic SEMG of biceps and interosseous muscles showed quite uniform frequencies, while tibialis's SEMG presented a more scattered frequency distribution. Therefore, the peak frequency was rather regular in biceps and interosseous myopathic SEMG signals, but variable in tibialis's SEMG, as exemplified in Fig. 9.

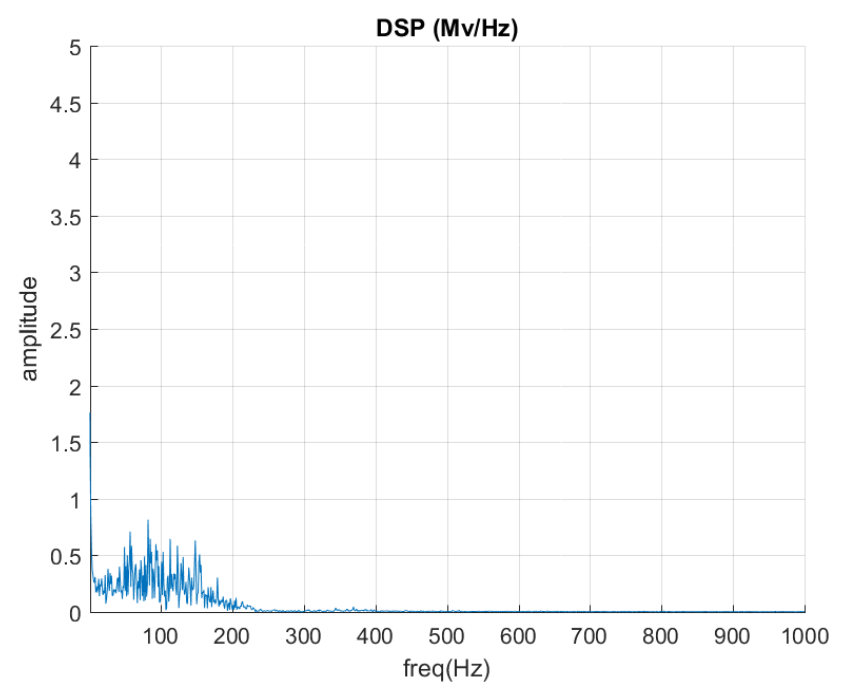

Fig. 7. PSD of Biceps SEMG Simulated Signal. 


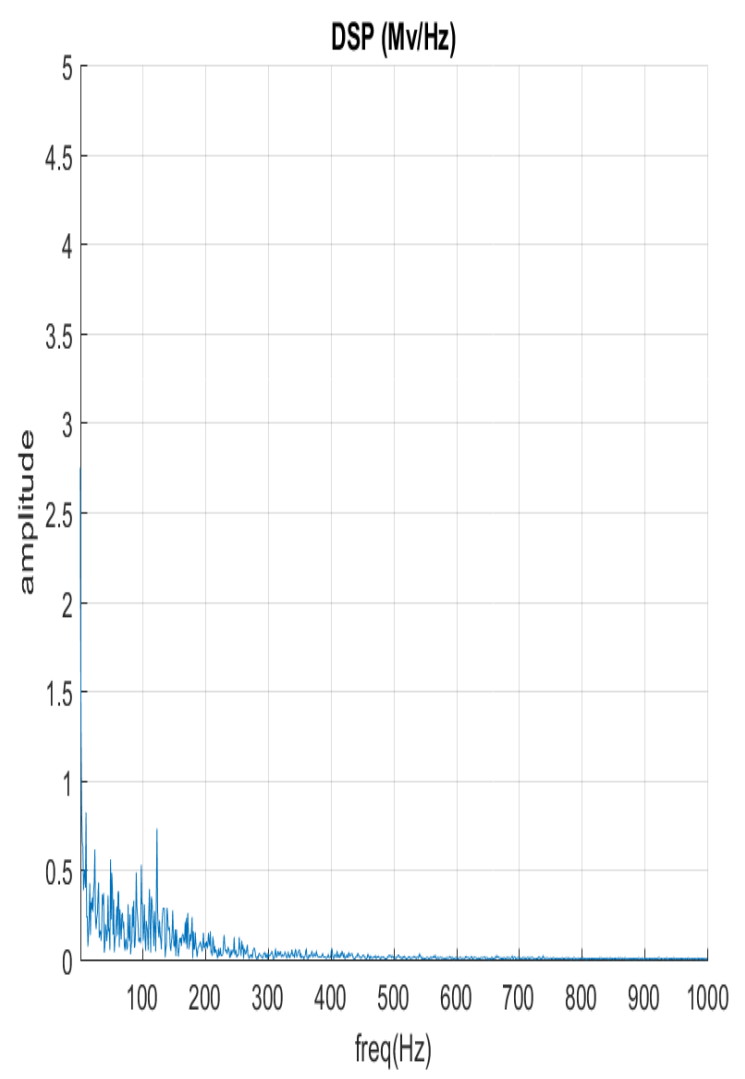

Fig. 8. PSD of Interosseus SEMG Simulated Signal.

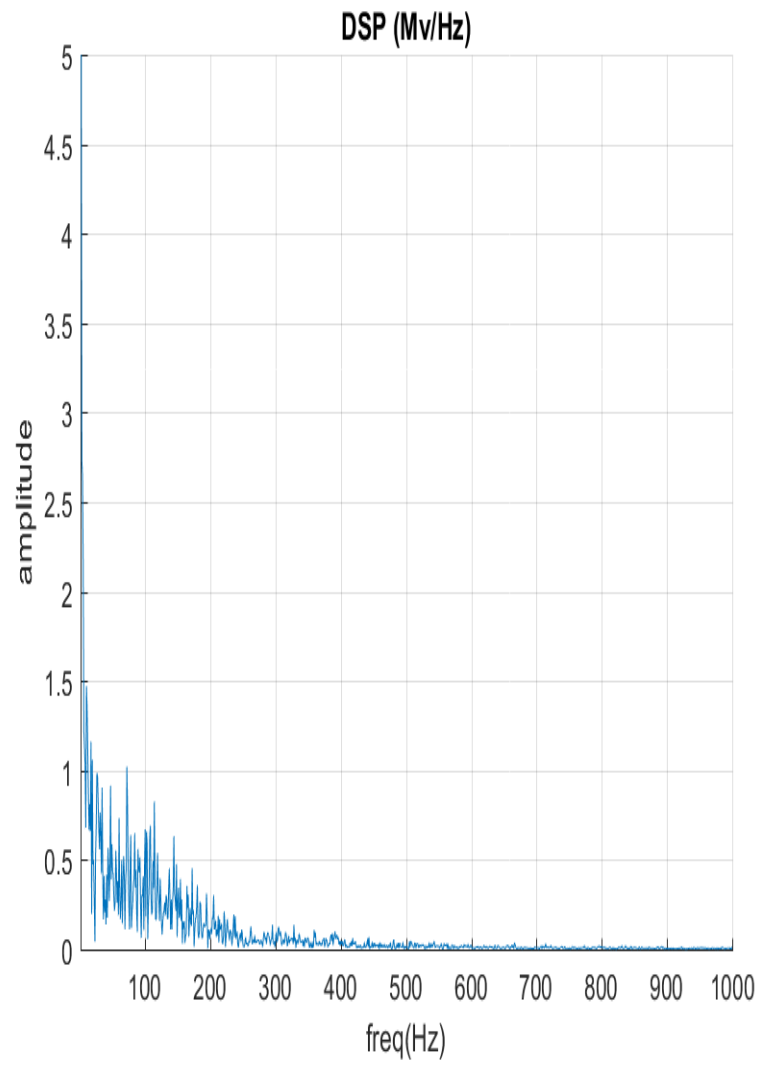

Fig. 9. PSD of Tibialis SEMG Simulated Signal.

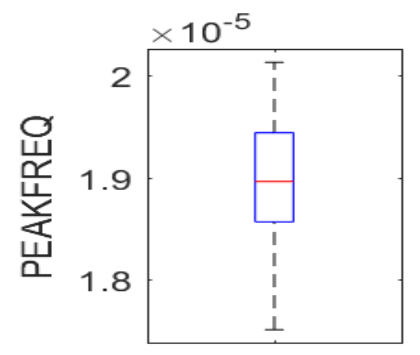

Biceps (myo)

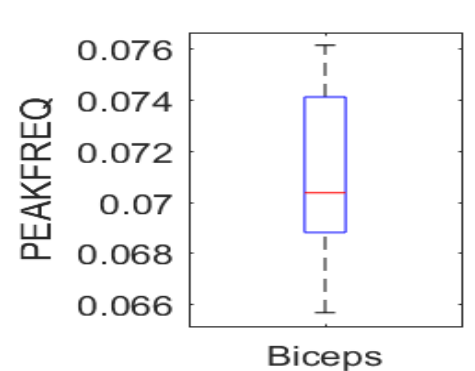

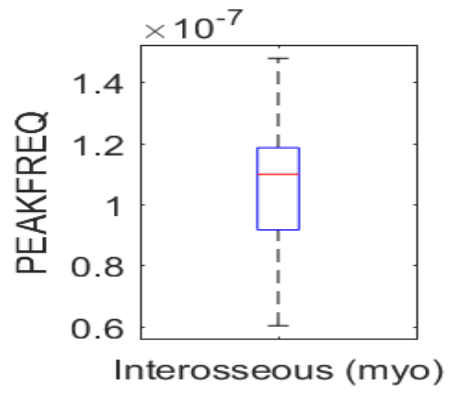
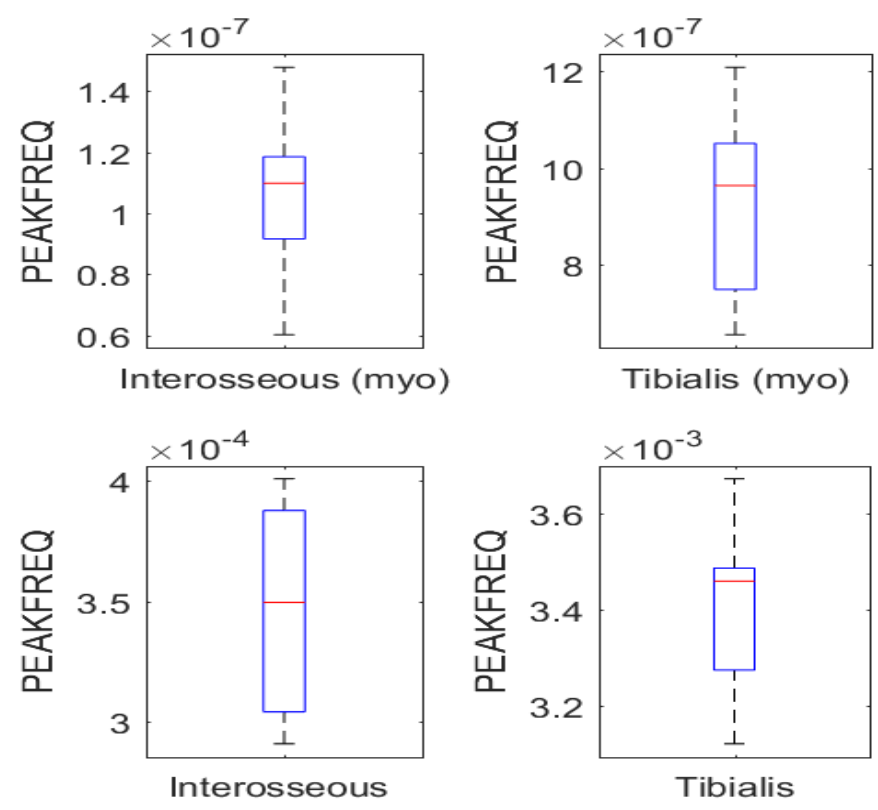

Fig. 10. Box plot of RMS. 


\section{CONCLUSIONS}

In this work, we presented a framework for simulating myopathic SEMG signals. It is composed of two main modules, the first one generates the MUAPs of each UM and the second one performs the spatiotemporally summing of the different MUAPs obtained from the first module. The obtained results allowed us to study the appearance and the characteristics of myopathic SEMG signals of different skeletal muscles such as: Biceps Brachii, Interosseurs Dorsalis and Tibalis Anterior. After the simulation of this kind of pathological signals, we conducted a comparative study of the synthetically recorded myopathic dataset with those normal. RMS and peak frequency of PSD where used to compare the synthetic results generated by our framework.

In our future work, different validation algorithms can be used to improve the performance of our framework and other type of disorder (like neurological disorder) can be integrated.

\section{REFERENCES}

[1] Robert Merletti and Dario Farina, editors. Surface Electromyography: Physiology, Engineering and Applications. John Wiley and Sons, Inc., Hoboken, New Jersey, USA., 2016.

[2] Andrew Hamilton-Wright, and Daniel W. Stashuk, Physiologically Based Simulation of Clinical EMG Signals. IEEE transactions on biomedical engineering, vol. 52, no. 2, february 2005

[3] K. C. McGill. Surface electromyogram signal modelling. Medical and Biological Engineering and Computing, 2004.

[4] Neville Hogan and Robert W. Mann. Myoelectric signal processing: Optimal estimation applied to electromyography - part I: Derivation of the optimal myoprocessor. IEEE Transactions on Biomedical Engineering, 27(7):382-395, July 1980.

[5] Eike Petersen and Philipp Rostalski. A Comprehensive Mathematical Model of Surface Electromyography and Force Generation. February 28, 2018

[6] Javier Rodriguez-Falces, Javier Navallas, and Armando Malanda. EMG modeling. In Ganesh R. Naik, editor, Computational Intelligence in Electromyography Analysis - A Perspective on Current Applications and Future Challenges. InTech, October 2012.
[7] Dario Farina, Luca Mesin, Simone Martina, and Roberto Merletti. A surface EMG generation model with multilayer cylindrical description of the volume conductor. IEEE Transactions on Biomedical Engineering, 2004.

[8] Mylena Mordhorst, Thomas Heidlauf, and Oliver Röhrle. Predicting electromyographic signals under realistic conditions using a multiscale chemo-electro-mechanical finite element model. Interface Focus, 5, 2015. doi: 10.1098/rsfs.2014.0076.

[9] D. Farina and R. Merletti. A novel approach for precise simulation of the EMG signal detected surface electrodes. IEEE Transactions on Biomedical Engineering, 2001.

[10] Lora A Major and Kelvin E Jones. Simulations of motor unit number estimation techniques. J. Neural Eng. 2 (2005) 17-34

[11] Jakob L. Dideriksen, Dario Farina, and Roger M. Enoka. Influence of fatigue on the simulated relation between the amplitude of the surface electromyogram and muscle force. Philosophical Transactions of the Royal Society A: Mathematical, Physical and Engineering Sciences, 2010.

[12] Luca, Carlo J., Alexander Adam, Robert Wotiz, L. Donald Gilmore, and S. Hamid Nawab. Decomposition of surface EMG signals. J Neurophysiol 96: 1646-1657, 2006; doi:10.1152/jn.00009.2006.

[13] George V. Dimitrov and Nonna A. Dimitrova. Precise and fast calculation of the motor unit potentials detected by a point and rectangular plate electrode. Medical Engineering and Physics, 1998.

[14] A. J. Fuglevand, D. A. Winter, and A. E. Patla. Models of recruitment and rate coding organization in motor-unit pools. Journal of Neurophysiology, 1993.

[15] Loredana R. Lo Conte, Roberto Merletti, and Guido V. Sandri. Hermite expansions of compact support waveforms: Applications to myoelectric signals. IEEE Transactions on Biomedical Engineering, 41(12):11471159, 1994.

[16] C. Sinderby, L. Lindström, and A. E. Grassino. Automatic assessment of electromyogram quality. Journal of Applied Physiology: Respiratory, Environmental and Exercise Physiology, 79(5):1803-1815, 1995.

[17] Van Veen, B. K., Wolters, H., Wallinga, W., Rutten, W. L., and Boom, H. B., The bioelectrical source in computing single muscle fiber action potentials. Biophysical Journal, 64(5):1492-1498, 1993.

[18] Wheeler KA, Kumar DK, Shimada H. An accurate bicep muscle model with sEMG and muscle force outputs. J Med Biol Eng.; 30(6):393-8, 2010. 